\title{
Self-assembled colloidal structures for photonics
}

\author{
Shin-Hyun Kim ${ }^{1}$, Su Yeon Lee ${ }^{2}$, Seung-Man Yang ${ }^{2 *}$ and Gi-Ra Yi ${ }^{* *}$ \\ Harvard University, USA, KAIST and Chungbuk National University, Korea
}

Colloidal self-assembly has been investigated as a promising and practical approach for the fabrication of photonic nanostructures, including colloidal crystals, composite and inverse opals, and photonic glasses. Depending on the interactions between the colloidal particles, colloidal structures can be affected dramatically and modulated by applying an additional external field. Furthermore, in contrast to other approaches, self-assembled nanostructures with large areas or designed shapes can be prepared at low cost. As a result, the use of such colloidal systems has been investigated in many practical photonic applications. In this review article, we describe the colloidal self-assembly of periodic and non-periodic photonic nanostructures in brief and then summarize recent achievements in the field of colloidal photonic nanostructures and their applications, which include displays, optical devices, photochemistry and biological sensors.

A s dielectric structures with a submicrometer length scale can interact strongly with light, various remarkable optical responses can be designed and tailored depending on the types and parameters of their structures. Over the past few decades, the unusual optical properties of the periodic dielectric structures called photonic crystals have been investigated intensively [1]. Many research groups have endeavored to engineer the optical properties of photonic crystals, including photonic bandgaps, 'slow' photons, negative refraction and other properties, or to use them in practical applications. Two-dimensional (2D) structures, which are mostly prepared by conventional lithographic processes, were demonstrated initially, in which total internal reflections were adopted for confining the light in a nonperiodic third direction, and their use has been investigated in some limited applications [2]. Three-dimensional (3D) structures have also been investigated intensively because of their complete photonic bandgaps in certain structures, a critical property for controlling light in $3 \mathrm{D}$ space. Research on such structures has been supported by the recent development of facile fabrication methods, including the selfassembly of simple monodisperse particles, also known as colloidal self-assembly [3], block copolymer self-assembly [4], the auto-cloning
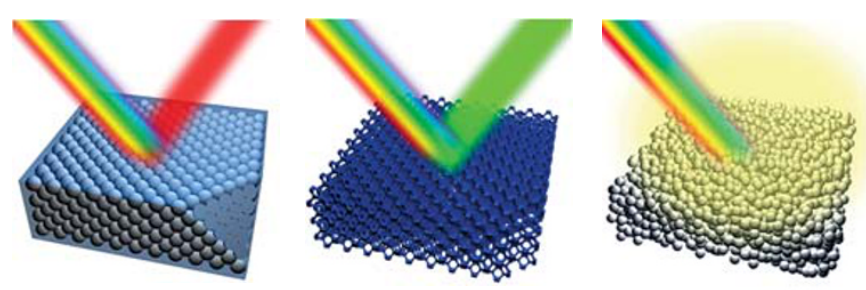

Figure 1. Colloidal crystals for photonics applications. (Left) Colloidal crystals can be formed into any shape to achieve desired reflectance properties. (Center) Inverse structures can be obtained by infiltrating the crystal with various materials and then removing the template particles (colloidal templating). (Right) Short-range-ordered structures of colloidal particles or colloidal glasses can be assembled to achieve angle-independent coherent optical scattering. process [5] and holographic lithography [6]. Of these methods, colloidal self-assembly is the most promising for the low-cost production of $2 \mathrm{D}$ and $3 \mathrm{D}$ photonic crystals over large areas or with various shapes $[7,8]$. Schematic diagrams of the basic colloidal crystal structure along with inverse and short-range-ordered scattering structures for photonic applications are shown in Figure 1. Disordered dielectric structures of monodisperse particles called photonic glasses have begun to be investigated as another class of photonic nanostructures that can manifest some unusual optical phenomena such as random lasing, strong light localization and long-range intensity correlations. In this review article, we describe self-assembled colloidal photonic nanostructures in brief and summarize recent achievements in the field of colloidal photonic nanostructures and their applications.

\section{Fabrication of photonic nanostructures by colloidal assembly}

\section{Colloidal crystals}

Since Vanderhoff's serendipitous discovery of a synthetic method for preparing monodisperse polymer colloids [9], the method has been extended to the preparation of a variety of polymeric colloids and also to the processing of inorganic colloidal particles such as silica, titania and iron oxide. As long as particles are stable in liquid and their size distribution is sufficiently narrow, they can be crystallized in a facecentered cubic ( $\mathrm{fcc}$ ) lattice by increasing their volume fraction through any concentration process, such as controlled evaporation, sedimentation or filtration. In general, the interparticle forces can be described by summing over the various potentials from different origins, including intermolecular forces, surface charges and steric stabilizers [10]. To produce stable particles, the attractive van der Waals force should be overcome by the strong long-range repulsive force, such as that due to steric or electrostatic repulsion. In the ideal case, the refractive indexes of the particle and medium are nearly matched, which makes the attractive force negligible and the repulsive force dominant. The Debye screening length $\left(\kappa^{-1}\right)$ is the characteristic length scale over which mobile charge carriers (e.g. electrons) screen out electric fields, and corresponds to the

\footnotetext{
*Corresponding authors. E-mail: smyang@kaist.ac.kr, yigira@chungbuk.ac.kr

' School of Engineering and Applied Science, Harvard University, Cambridge, MA 02138, USA

2 Department of Chemical and Biomolecular Engineering, KAIST, Yuseong-gu, Daejeon 305-701, Korea

${ }^{3}$ Department of Engineering Chemistry, Chungbuk National University, Heungduk-gu, Cheongju Chungbuk 361-763, Korea
} 

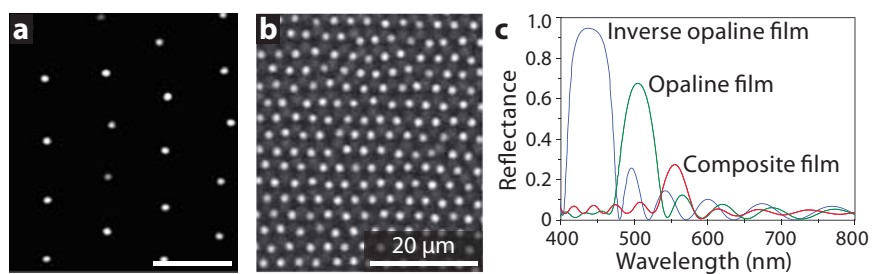

Figure 2. Optical properties of colloidal crystals. $(a, b)$ Confocal microscopy images showing the crystalline structure of $2 \mu \mathrm{m}$ PMMA particles stabilized with poly(12hydroxystearic acid) and crystallized in (a) cyclohexyl bromide (CHB), forming a bodycentered cubic lattice with an interparticle distance of $\sim 17 \mu \mathrm{m}$, and (b) a mixture of decalin and $\mathrm{CHB}$, forming a face-centered cubic lattice. Adapted from Ref. 11 (๔ 2003 IOP). (c) Reflectance profiles of an opaline film, composite film and inverse opaline film with 12 layers of spheres in the (111) direction, as predicted by finite-difference time-domain simulations for a matrix with refractive index of 1.6 and silica spheres with diameter of $230 \mathrm{~nm}$ and refractive index of 1.45

strength of the electrostatic repulsion force [10]. In the case of colloidal systems, $\kappa^{-1}$ is defined by

$$
\kappa^{-1}=\sqrt{\frac{\varepsilon_{\mathrm{r}} \varepsilon_{0} k_{\mathrm{B}} T}{2 N_{\mathrm{A}} e^{2} I}}
$$

where $I$ is the ionic strength of the electrolyte $\left(\mathrm{mol} \mathrm{m}^{-3}\right), \varepsilon_{0}$ is the permittivity of free space, $\varepsilon_{\mathrm{r}}$ is the dielectric constant, $k_{\mathrm{B}}$ is the Boltzmann constant, $T$ is absolute temperature, $N_{\mathrm{A}}$ is the Avogadro number and $e$ is elementary charge.

In the case of a system in which density and refractive index are matched, as shown in Figure 2(a), in which $\kappa^{-1}$ for polymethylmethacrylate spheres in cyclohexyl bromide $(\mathrm{CHB})$ is greater than the particle size, a long-range ordered crystalline structure is formed. On the other hand, the electrostatic repulsion force can be screened in a controlled manner by adding a nonpolar solvent, decalin, which results in shortened interparticle distances in colloidal crystals (Figure 2(b)) [11]. Furthermore, by applying an external electric or magnetic field, the richer crystalline phases of colloids could be explored in a controlled manner [12]. This experimental approach has recently been extended to other ordinary materials for practical applications. For instance, highly stable colloidal crystals have been permanently trapped in crosslinked polymer hydrogels as a basis for the development of color-changing sensors and lasers [13-16]. More recently, polymerizable liquids have been introduced as media for colloidal crystals. When the refractive indexes of such media and the silica particles are nearly matched, repulsion force between the particles become dominant, which results in colloidal structures with long-range order. To control the overall shape of composite colloidal crystals, doctor-blade coating and spin-coating processes are used for flat films [17,18], and confined geometries have been adapted for other shapes such as spheres, ellipsoids, fibers and lined structures [19]. Formation processes involving dispersion in crosslinkable or polymerizable liquid and solidification are much faster than conventional crystallization processes such as evaporation, sedimentation, filtration or dip-coating from colloidal solution in evaporable liquids [20-23].

\section{Inverse opals}

By infiltrating the interstices of polymer latex colloidal crystals with inorganic materials and subsequently burning out the polymer latex, ordered macroporous films known as 'inverse opals' can be prepared (Figure 1). Inverse opals have higher reflectivities over wider optical stop gaps due to the higher refractive index mismatch between the spheres and the medium. It is known that these structures can have $3 \mathrm{D}$ photonic bandgaps in the very low-wavelength or high-frequency regions. Although their bandgaps are very narrow and can be closed even by small defects, they exhibit wider stop gaps and higher mechanical stability. In the production of inverse opal structures, colloidal crystals of inorganic colloids (e.g. silica) can also be used, in which composites with polymeric materials are formed and then converted to polymeric inverse opal structures by removal of the inorganic particles using selective etchants such as hydrofluoric acid. In addition, colloidal crystals of polymeric particles in which the interstices are filled with highly crosslinked polymers can be etched out through the use of a selective organic solvent when the matrix is crosslinked but the spheres are not.

During the formation of an opal or an inorganic matrix, the capillary force between particles or the volume shrinkage of the inorganic matrix during reaction can cause the spontaneous formation of significant numbers of cracks in the opaline structure. To avoid this problem, the colloidal particles can be dispersed in polymerizable liquids, as mentioned in the previous section, or be co-assembled with small inorganic nanoparticles or precursors [24]. Cracks form in co-assembled systems only for films thicker than a critical value. Furthermore, when a photocurable liquid is used for the matrix, a variety of patterns and structures of composite colloidal crystals can be fabricated, and the inverse structures can be produced by using an appropriate etching technique.

The optical properties of these structures can be predicted through finite-difference time-domain simulations. Optical reflectivity calculations for opal, composite and inverse opal structures derived from silica spheres with a diameter of $230 \mathrm{~nm}$ and a refractive index of 1.45 densely packed into 12 layers of an fcc lattice on a flat hexagonally packed (111) plane are shown in Figure 2(c). The reflectance peaks $(\lambda)$ are produced by the photonic bandgap, and the background wavy spectra are attributed to Fabry-Pérot interference in the thin film, that is, interference between the multiple reflections of light between the two reflecting surfaces (air-film and air-substrate interfaces) [25].

The reflectance peaks for the (111) plane of an fcc lattice can be determined simply by Bragg's law, as follows:

$$
\lambda=2 d n_{\mathrm{eff}}=\left(\frac{\pi}{3 \sqrt{2} \phi}\right)^{1 / 3}\left(\frac{8}{3}\right)^{1 / 2} D\left(n_{\mathrm{p}}^{2} \phi+n_{\mathrm{m}}^{2}[1-\phi]\right)^{1 / 2}
$$

where $d$ is the lattice spacing, $n_{\text {eff }}$ is the effective refractive index, $D$ is particle diameter, $\phi$ is particle volume fraction, and $n_{\mathrm{p}}$ and $n_{\mathrm{m}}$ are the refractive indexes of the particles and the medium. In addition, the Bragg attenuation length of the colloidal crystal, which is a measure of the exponential decay of evanescent waves inside the gap [26], is roughly inversely proportional to the index contrast. The full-width at halfmaximum $(\Delta \lambda)$ or wavelength gap can be estimated by

$$
\frac{\Delta \lambda}{\lambda} \cong \frac{\left|n_{\mathrm{p}}-n_{\mathrm{m}}\right|}{n_{\mathrm{eff}}}
$$

Using these equations, we can interpret Figure 2(c) and predict the reflectance spectrum of an arbitrary photonic crystal. Further information on the diffraction phenomena in ordered colloidal structures, such as angles, intensities and bandwidths, can be predicted and understood using dynamical diffraction theory $[27,28]$.

\section{Photonic glasses}

Noncrystalline monodisperse colloid structures or photonic glasses, which are essentially random close packings of monodisperse colloids, have recently been reported. As such structures are actually aggregates of monodisperse colloids, they have short-range order, which is essentially order over a range of a few particles and which can be detected from the structure's diffraction pattern [29]. Whereas photonic crystals can be used in the precise manipulation of ballistic photons, photonic glasses are useful in the control of light diffusion. The random structures of designed uniform colloids can interact strongly with light and thus produce unusual diffusion phenomena including random lasing, angle-independent color and light localization [30-36]. These disordered monodisperse colloidal particle structures and short-rangeordered colloidal structures have been produced by controlling the salt 
a

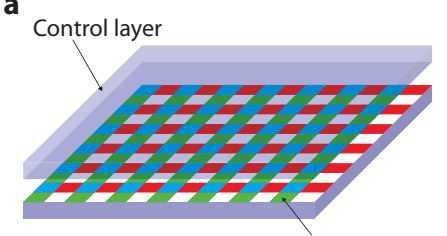

RGBW pattern

c

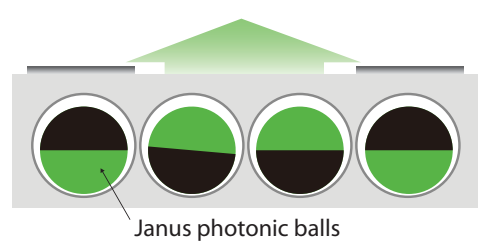

b

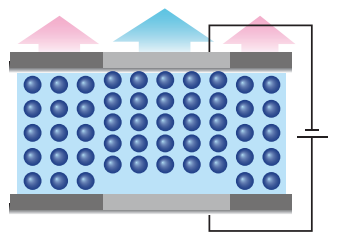

d

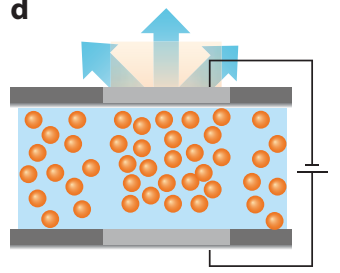

Figure 3. Schematic summary of display devices based on colloidal-structured photonics. (a) Passive-mode display with pixelated photonic crystals. (b) Active-mode display based on dynamic modulation of lattice constant. (c) Gyricon display based on rotation of Janus photonic microspheres. (d) Display device with isotropic colloidal structure of short-range order for angle-independent structural color.

concentration in order to destabilize the colloidal particles or by adding smaller colloidal particles [37].

\section{Selected applications}

A variety of applications have been investigated for the four types of photonic nanostructures mentioned above. In this section, several important applications of photonic nanostructures fabricated via colloidal assembly are discussed, ranging from displays to solar cells and sensors.

\section{Structural color-based display devices}

The regular lattices of colloidal crystals exhibit reflection colors produced by light interference, such as the beautiful metallic luster observed in opals, that cannot be achieved by transmission using optical filters. To distinguish these colors from chemical colors based on the absorption spectra of chemicals, they are referred to as structural colors. If structural colors are used in a display, viewers can see the display clearly even under the high intensity of daytime light. In addition, energy consumption

can be reduced significantly because such devices use ambient light as the light source without backlighting. Therefore, many researchers have developed colloidal crystal-based color pigments for various types of display devices ranging from portable devices to outdoor signboards or large billboards. Three different approaches have been studied: pixelation of colloidal crystals as an alternative to conventional optical filter patterns [38], dynamic tuning of reflection colors by controlling the lattice constants of photonic crystals [39] and rotation-based displays comprised of photonic microparticles with anisotropic optical properties [40]. In Figure 3, we summarize these three types of display devices based on colloidal crystals. The figure also shows the low angle-dependence of structural color for a display device based on a short-rangeordered colloidal structure [41].

Pixelated photonic crystal films capable of producing red, green, blue and white can be used directly in liquid-crystal display (LCD) devices as replacements for the color filter and backlight units (Figure 3(a)). As liquid crystal makes it possible to control the reflection intensity for each color pixel as in conventional LCDs, color and brightness can be realized by the pixelated micropatterns of photonic crystals. However, the pixelation of colloidal crystals into $2 \mathrm{D}$ space is challenging. The first attempt to pattern colloidal crystals involved use of the micromolding in capillaries (MIMIC) technique [42]. By this approach, microcapillaries are filled with a colloidal suspension, which forms crystals during directional drying from the ends of the capillaries. Colloidal crystal patterns of various shapes, including lines and stars, can be produced by varying the micromolds, which are prepared by soft lithography. Further, colloidal crystals with two different bandgap positions can be patterned through a combination of MIMIC and the vertical deposition of colloids onto microchannels, as illustrated in Figure 4(a) [43]. When the interstices of the MIMIC-assisted colloidal crystals are filled with polymers, the resulting colloidal crystal patterns can be used as a template for the selective deposition of colloidal crystals into the empty spaces between the patterns by using a dip-coating process. As a result, two distinct domains of colloidal crystals can be produced in a desired shape. Recently, colloidal crystals with 20 different bandgaps were integrated into a single film by employing MIMIC on a photocurable suspension of repulsive colloids to produce an optical spectrometer [44]. However, MIMICbased approaches have intrinsic limitations in pattern shape and production rate, which make pixelation impossible.

A higher degree of freedom in patterning can be achieved through a photolithography-based approach. Various clever techniques have been

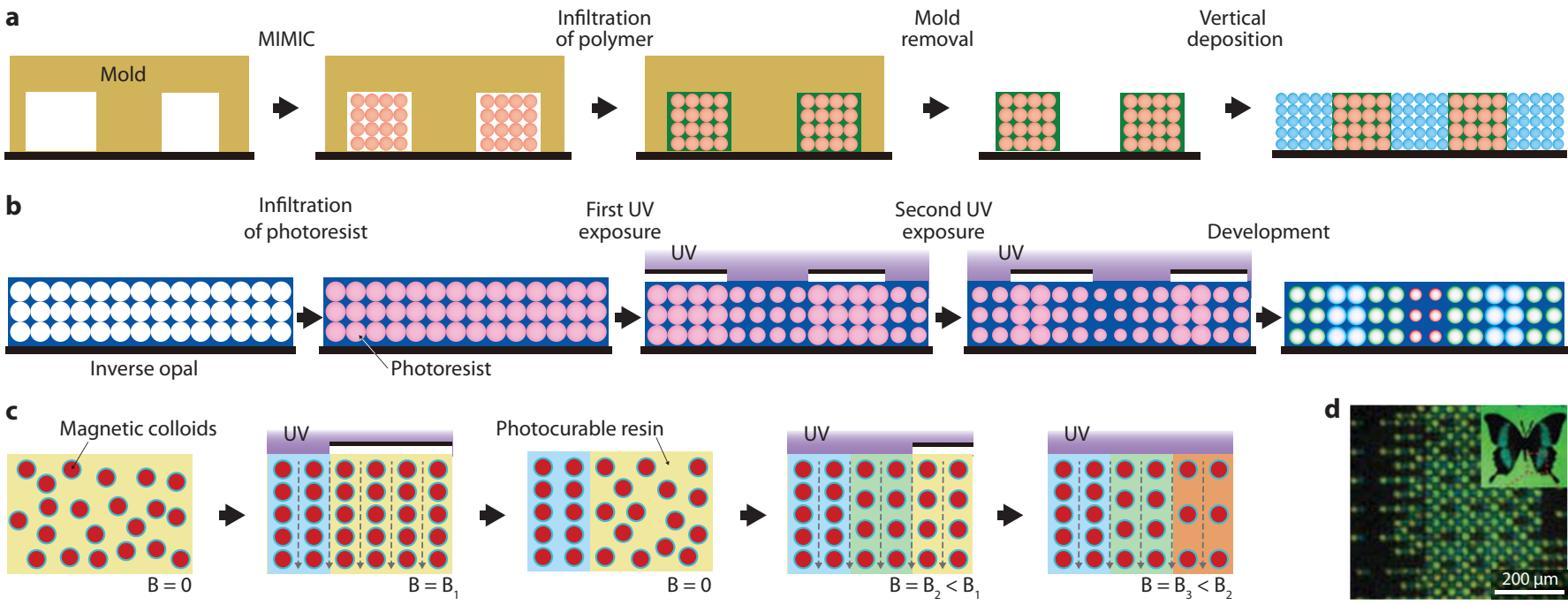

Figure 4. Patterning of photonic crystals. (a) Patterning process for two different colloidal crystals by combining the MIMIC technique and vertical deposition. (b) The pixelation method for red, green and blue (RGB) colors. Inverse opal with pores filled with SU-8 is exposed twice under UV light via a line-pattern photomask at $0^{\circ}$ and $90^{\circ}$. (c) Patterning of one-dimensional photonic crystals of colloidal chain-like structures. Superparamagnetic particles are aligned into chains under an external magnetic field. Selective localized UV exposure then fixes the structure. (d) Optical microscopy image of blue and yellow dot patterns forming the butterfly image inset. Modified after Ref. 47. 
developed for the preparation of patterns by selective ultraviolet (UV) exposure via photomasks. Substrates with wettability that can be controlled by adjusting the UV dosing were first used to selectively deposit colloids onto the hydrophilic area of the substrate [45]. A hydrophobic surface composed of fluoroalkylsilanes on a titania film was found to exhibit a transition in water drop contact angle from $100^{\circ}$ to $0^{\circ}$ as a result of UV exposure owing to the decomposition of fluoroalkylsilane and the creation of surface oxygen vacancies through the photocatalytic effects of titania. When this substrate was employed in the vertical deposition of an aqueous colloidal suspension, the area exposed to UV was selectively coated with colloidal crystals. The additional steps of fluoridation and selective UV exposure thus enabled the fabrication of a pattern with the desired colloidal crystal shape and two different bandgaps. Simpler methods based on photoresists have since been developed to achieve higher resolution. The spin-coating of photocurable suspensions onto wafers induces the crystallization of colloids by shearing, and a subsequent photolithographic process can then be used to create a pattern of colloidal crystals [18]. In addition, the infiltration of photoresists into the interstices of colloidal crystals can be used to prepare patterns with smaller features [46]. However, these methods require complex and fragile procedures including the multi-step crystallization of colloids and photomask alignment to obtain multicolor patterns.

One clever and simple scheme for the preparation of pixels of colloidal crystals with three different colors was developed by Lee et al. [38]. As illustrated in Figure 4(b), this method uses inverse opal films with air pockets filled with the photoresist SU-8. By exposing the inverse opal to UV via a line pattern photomask at one orientation and then rotated by $90^{\circ}$, three different regions were prepared: an unexposed region, a onceexposed region, and a twice-exposed region. As the UV dose affects the degree of polymerization of SU-8, the three regions had different pore sizes after the development of uncrosslinked SU-8. Using this approach, it was possible to pixelate three distinct inverse opal structures with different bandgap positions through single-step crystallization without the alignment of photomasks. This type of pixelated inverse opal film can be used directly as a color reflector in a microdisplay device by capping with a liquid-crystal control layer.

Recent advances in photolithography-based techniques have made it possible to fabricate colloidal photonic crystal patterns in real time [47]. When an external magnetic field is applied to superparamagnetic colloidal particles dispersed in a photocurable medium, the particles form a one-dimensional chain-like structure that exhibits structural color. As the particles repulse each other due to electrostatic and solvation forces, the intensity of the magnetic field determines the interparticle distance. A solvation force arises due to the formation of a dense solvation layer via the hydrogen bonding of solvent molecules onto the particle surface. Photonic chain structures can also be fixed in a specific area by selective UV exposure using a digital micromirror array. The combination of realtime manipulation of the exposure pattern and control of the magnetic field intensity makes it possible to produce any type of color pattern, as shown schematically in Figure 4(c). Of particular interest in this context is the demonstration that color mixing and reflectivity control can be achieved through the spatial distribution of dots of two different colors. The butterfly wing in the inset of Figure $4(\mathrm{~d})$ is composed of numerous pixels of two different colors.

Patterning has also been studied for dome-shaped colloidal crystals. Small suspension droplets patterned with ink-jet or dispenser machines can be used to produce hemispherical colloidal crystals with (111) planes aligned along the curved interface. Through drying or photopolymerization of the patterned droplets, single- or multicolored photonic domes with rotation-independent isotropic structural color can be patterned [48-51]. Note that photonic domes can also be patterned by selective droplet adhesion onto prepatterned substrates without requiring the one-by-one deposition of droplets [52,53].

Dynamic tuning of the photonic bandgap of colloidal crystals enables the fabrication of display devices without the need for pixelation or liquid-crystal units (Figure 3(b)). Magnetic and electric fields

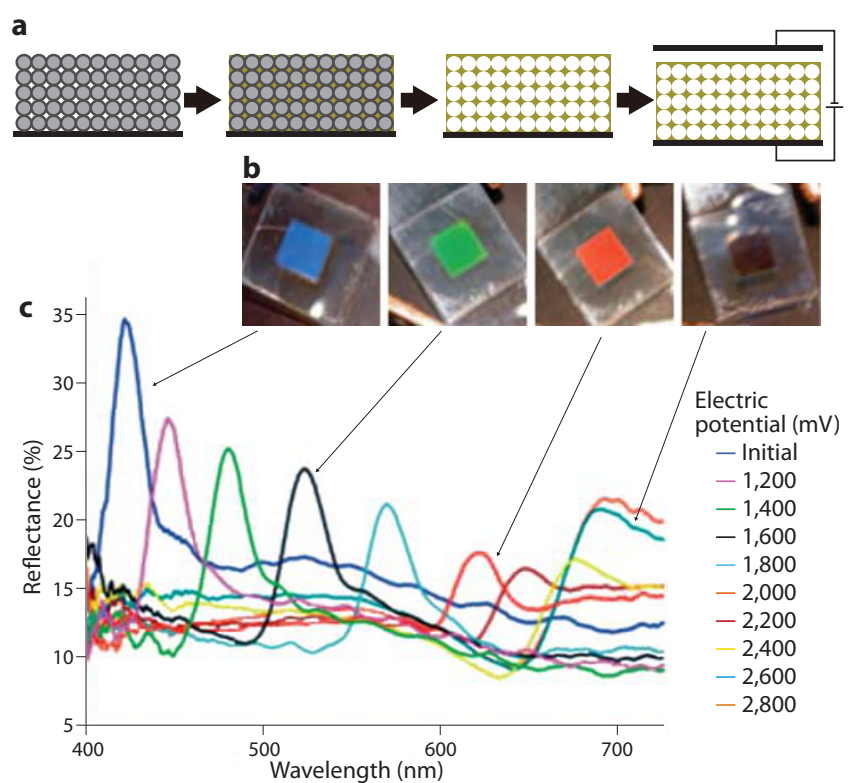

Figure 5. Dynamic modulation of the photonic bandgap. (a) Schematic diagram of the preparation of an electroresponsive inverse opal film. (b) Optical images and (c) reflectance spectra of an inverse opal film for a range of electric potential after $10 \mathrm{~s}$. Adapted from Ref. 57 (๔ 2009 Wiley-VCH).

are considered to be the optimal stimuli for modulation of the lattice constants of colloidal crystals and inverse opals for display applications. Superparamagnetic colloidal particles dispersed in an aqueous medium form non-close-packed colloidal crystals with an fcc lattice, a structure known as a crystalline colloidal array (CCA), due to surface charge-induced electrostatic repulsion. When an external magnetic field is applied to such an array, the lattice is compressed along the field direction by the attractive magnetic force, resulting in a blue-shift of the structural color, where the degree of compression is determined by the intensity of the magnetic field [54]. In addition, all visible colors from red to violet can be prepared by increasing the loading of magnetic materials in the colloids. The switching of color in such systems is very rapid, requiring only $200 \mathrm{~ms}$ [55]. In an alternative approach, dynamic tuning of the structural color of superparamagnetic particles can be achieved in nonaqueous solvents, which has several advantages, including nonvolatility, for applications in display devices [56]. Although the electrostatic repulsion between particles is diminished in nonaqueous solvents, repulsive forces can arise due to solvation as discussed above. The particles exist in a random arrangement in the absence of a magnetic field, and become organized into a chain-like structure when an external field is applied. Such magnetic field-induced modulation of the structural color provides fast response and full color tuning, however the local tuning of color on the micrometer scale is difficult to achieve, which limits the range of applications of this approach.

Electric field-induced control of structural color is favored because of the local tuning of color and the relatively simple integration of such a mechanism into devices. Pioneering studies of the electrical modulation of the photonic band position were performed by Ozin and co-workers. Using silica opals with interstices filled with an electro-responsive polymeric matrix, they manipulated the swelling and deswelling of the matrix and therefore the red and blue shifts of colors [39]. When the matrix polymer, polyferrocenylsilane (PFS), is immersed in an electrolyte medium, it swells at oxidative potentials higher than the redox potential of PFS through diffusion of the anions and solvent from the electrolyte into the polymer to neutralize the positive charge buildup caused by the removal of electrons from the iron atoms of PFS. Under a reducing potential, this process reverses and the deswelling of the polymer and therefore a blue-shift of the color are observed. Further, the performance of the device can be enhanced by removing silica particles 
from the composite, which facilitates the transport of ions through large pores [57]. A scheme for the preparation of electroactive inverse opals is shown in Figure 5(a). Through the application of electric potentials up to just $2.8 \mathrm{~V}$, the full visible range of color can be covered with a single inverse opal film, as shown in the images and spectra in Figures 5(b,c). In addition, the color switching time is as short as a few seconds, which is a very short time for a swelling-based system. Ueno et al. [58] reported electrically tunable inverse opals composed of a polyelectrolyte gel immersed in a salt-free organic solvent. The electric dragging force on the immobilized charges in the polyelectrolyte matrix induces an anisotropic deformation of the matrix in the electric field direction, which enables tuning of the structural color of the inverse opals.

Recently, Shim et al. [59] reported the dynamic modulation of the photonic bandgaps of CCAs in an aqueous medium by varying the electric field. When the external electric field is applied to the CCA, the top area of the lattice is compressed or relaxed depending on the direction of the field due to the electrokinetic force on the particles. This system is based on the movement of particles rather than on the swelling of the matrix, so the switching time is reduced to $50 \mathrm{~ms}$. In addition, local tuning of the color was demonstrated using patterned indium tin oxide-coated glasses. However, there is a problem related to the evaporation of water and so this medium should be replaced with a nonvolatile solvent for long-term use.

Photonic microparticles with anisotropic optical characteristics have also been prepared for use in structural color-based display devices (Figure 3(c)). These Janus microparticles, composed of colloidal crystals and a carbon black-loaded polymeric matrix in each hemisphere, were fabricated using a microfluidic device and can be used as display pigments [40]. Their anisotropic electrical properties, due to the uneven distribution of carbon black, enable the microparticles to be aligned under an external electric field. Contrast is generated by orienting the colloidal crystal side up in local regions and the carbon black side up in the remaining regions. In an alternative approach, Ge et al. [60] produced anisotropic microspheres by solidifying emulsion droplets of a photocurable superparamagnetic particle suspension under a magnetic field. The magnetic particles in this system are aligned into a chain-like structure under a magnetic field and then fixed into polymeric spheres by polymerization. As a result, rotation of the microspheres can be achieved by the application of an external magnetic field, which switches the structural color.

Although many structural color-based display devices have been reported, this area of research still faces many challenges. Reducing the angle dependence of the diffraction color is particularly important in order to obtain wide display viewing angles. One promising strategy for achieving this involves using the isotropic structure of short-range order on the length scales of wavelengths, an approach that provides noniridescent colors that are invariant with respect to the viewing angle under omnidirectional lighting [61]. Recently, Lee et al. [41] demonstrated a tunable full color display device with no viewing angle dependence using electroresponsive non-periodic colloidal structures (Figure 3(d)). The introduction of nanoparticles into a matrix of composite colloidal crystals has also been effective for reducing the viewing angle dependence [62]. However, achievement of both low viewing angle dependence and high reflection intensity remains a challenge. Structural or alignment modifications of such crystal lattices or the development of colloidal crystal structures with new phases have the potential to provide wider viewing angles as well as high reflectivity. Faster switching and a wider tunable color range should also be addressed in order to facilitate the application of colloidal structures in practical display devices. The development of field-responsive colloidal particles that form photonic structures in nonvolatile liquid is one of the promising approaches to achieve fast and wide color switching simultaneously.

\section{Optical devices}

Research into photonic devices with colloidal structures has been relatively inactive in comparison with the use of slab-type $2 \mathrm{D}$ photonic
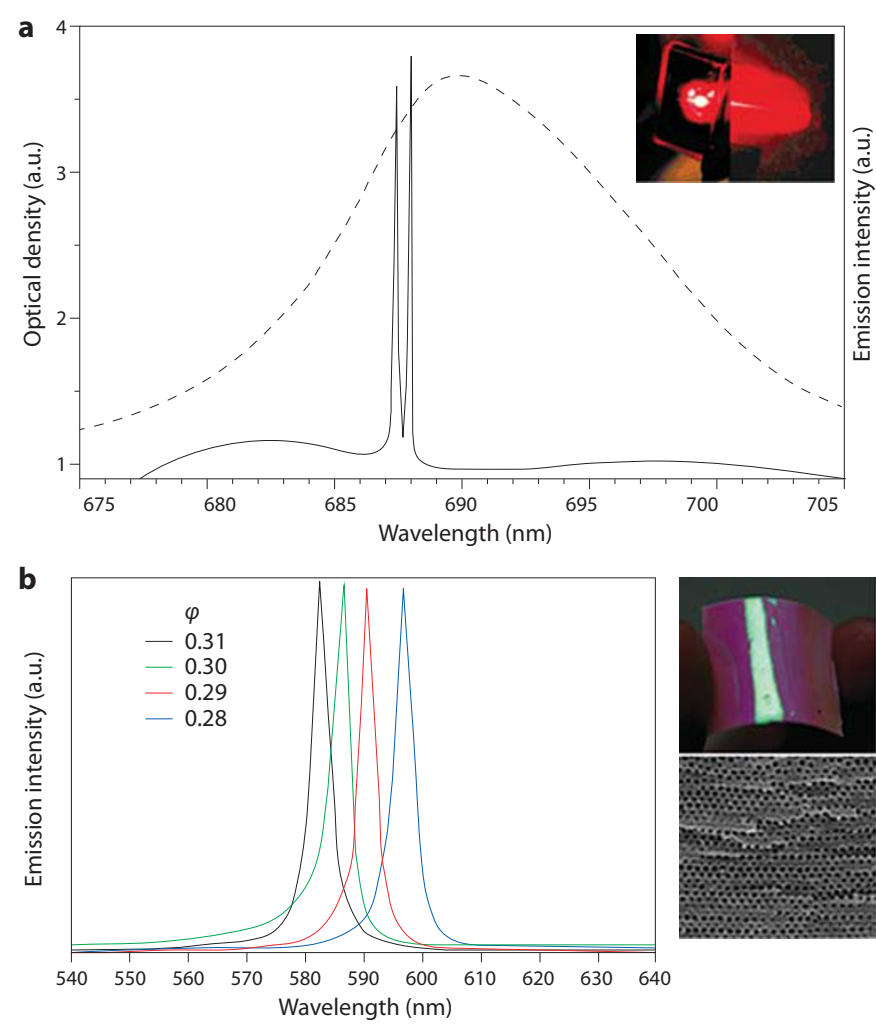

Figure 6. Lasers based on colloidal crystals. (a) Optical density spectrum for an opal immersed in methanol containing red oxazine dye molecules (left axis, dashed line), and the corresponding output spectrum of the (111) plane of the opal under optical pumping (right axis, solid line). Inset shows the lasing action of the opal. Adapted from Ref. 66 (๔ 2002 Wiley-VCH). (b) Output spectra of porous films prepared from four different volume fractions $(\varphi)$ of colloids in a photocurable medium. Optical and cross-sectional scanning electron microscopy images of a porous photonic film are shown right. Adapted from Ref. 68 (๔ 2009 ACS).

crystals for such applications. Although colloidal crystals can be used to achieve periodic modulation of the refractive index in $3 \mathrm{D}$ space, the crystal structures of spontaneously organized assemblies are limited to fcc, hexagonal close-packed and body-centered cubic lattices, which produce a stop band for a specific crystal direction rather than a full bandgap for all directions. Recent progress in the crystallization of charged colloidal particles has enabled the creation of new crystal phases [63-65]. However, large-area production with low defect density is required in order to realize the practical use of these new phases. As a result, most research into photonic devices has involved use of the stop bands of fcc structures.

One of the pioneering studies on photonic devices based on colloidal structures was performed by Shkunov et al. [66], who reported colloidal photonic crystal lasers constructed using silica opals. In that study, an opal with millimeter-scale thickness was prepared by sedimentation and then immersed in a liquid medium containing dissolved dye. When the spontaneous emission spectrum of the dye overlaps the band-edge position of the colloidal crystals, a directional laser beam is generated under optical pumping, as shown in Figure 6(a). This beam generation is caused by stimulated emission at the band edge, where the photon density of states is high and the group velocity of light is low. An opal immersed in two different dyes can produce two different wavelengths of lasing depending on the crystal direction, corresponding to two distinct band-edge positions. After the first report of lasing in colloidal crystals, such resonators have been improved by several research groups. Yamada et al. [67] reported lasing at the band-edge position of a polymerized CCA (PCCA) composed of mesoporous silica particles and a polymeric gel matrix swollen by an aqueous solution of dye molecules. Recently, Kim et al. [68] prepared porous photonic films doped with dye molecules, in 

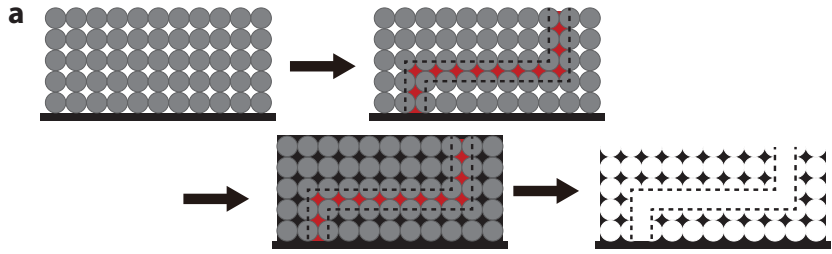

b

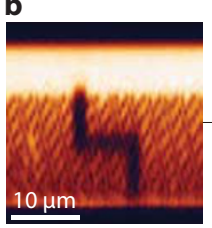
C

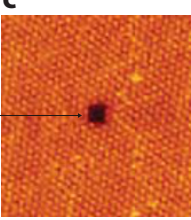

d

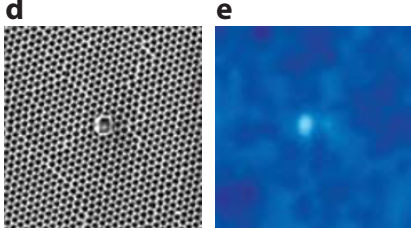

Figure 7. Waveguide embedded in a 3D photonic crystal with a full bandgap. (a) Preparation of the silicon inverse opal structure containing the waveguide. The waveguide template was prepared by two-photon polymerization. (b) Cross-sectional confocal microscopy image of the waveguide structure and corresponding (c) confocal, (d) scanning electron microscopy and (e) infrared microscopy images of one end of the waveguide. The light detected in (e) is propagated from the other end of the waveguide. Adapted from Ref. 74

which non-close-packed crystals of silica particles were used as a sacrificial template. As these porous films have a larger refractive index contrast than previous opals or PCCAs, it is possible to reduce the thickness of the liquid-free resonator to tens of micrometers. In addition, as shown in Figure 6(b), the lasing wavelength can be tuned by varying the lattice constant, which is determined by the volume fraction of particles before particle removal. In an alternative approach, Scharrer et al. [69] fabricated a zinc oxide inverse opal structure for the lasing of UV light in high-order bands without dye molecules; in this structure zinc oxide acts as a gain medium as well as the backbone of the photonic crystal.

Lasing with colloidal photonic crystals can also be achieved in defect mode. Furumi et al. [70] prepared colloidal crystals containing planar defects, in which each polystyrene colloidal crystal is infiltrated with polydimethylsiloxane and the defect layer is gel-doped with dye molecules. These films are highly flexible and exhibit laser emission at a wavelength corresponding not to the band edge but to the defect mode. In another approach, Lawrence et al. [71] used a PCCA as a tunable partial mirror in a Fabry-Pérot cavity. The confinement of the gain medium between the PCCA and a dielectric stack (perfect mirror) gives rise to lasing at a bandgap wavelength that can be tuned by compressing the PCCA.

Colloids with disordered random structures have been found to lase in a manner different to that of photonic crystals. Without photonic bandgap properties, the multiple scattering from disordered colloids with gain media can give rise to stimulated emission with the typical threshold behavior of lasers. However, the wavelength of the laser is determined by the maximum of the gain spectrum and the lasing direction is uncontrolled [35]. Recent progress in random lasers has made it possible to tailor the wavelength of laser emission by employing photonic glasses containing a completely disordered arrangement of monodisperse colloids [30,37]. Depending on the size and refractive index of the constitutive colloids, the Mie resonance modes are modulated and so the wavelength of the random laser can be shifted from the maximum of the gain spectrum.

Inverse opals can exhibit a full bandgap between the eighth and ninth energy bands. However, precise fabrication techniques and a high index contrast of at least 1.85 are required to open the full bandgap. Even though the bandgap is fragile with respect to even low defect densities due to the narrow bandwidth, silicon inverse opal structures with a full bandgap have been successfully prepared [72,73], and Rinne et al. [74] created waveguides embedded in silicon inverse opals with a full bandgap. As shown in Figure 7(a), two-photon polymerization can be used to fabricate a waveguide structure in a colloidal crystal. This process enables the selective polymerization of the monomer in the desired $3 \mathrm{D}$ region.
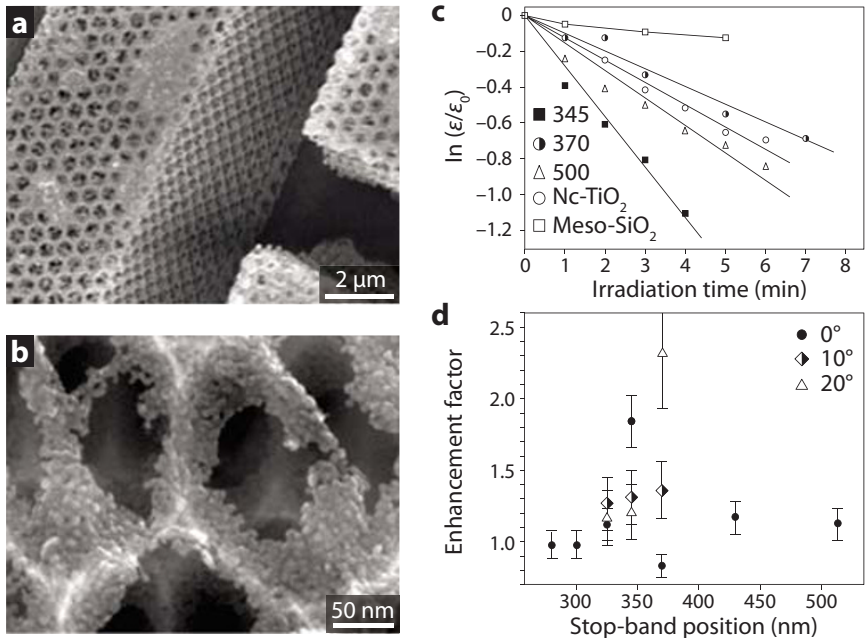

Figure 8. Enhancement of photochemical reaction efficiency by the effect of slow photons. (a,b) Scanning electron microscopy images of an inverse opal structure composed of titania nanocrystallites. (c) Degradation of methylene blue molecules adsorbed in three different inverse opals with bandgaps of 345, 370 and $500 \mathrm{~nm}$ according to the time of irradiation with monochromatic light centered at $370 \mathrm{~nm}$. The results for methylene blue adsorbed in nanocrystalline (NC) $\mathrm{TiO}_{2}$ powder and mesocrystalline $\mathrm{SiO}_{2}$ are shown for reference (control and blank, respectively). (d) Angle dependence of enhancement factor for inverse opals with various bandgap positions. Adapted from Ref. 75 (๔ 2006 Wiley-VCH)

Three-dimensional full-bandgap materials with an embedded waveguide (Figure 7) can be obtained by chemical vapor deposition of amorphous silicon and selective removal of the silica and the polymeric waveguide template. Although large losses occur during propagation through this waveguide structure, this research is significant because it is the first reported fabrication of a photonic device with $3 \mathrm{D}$ full-bandgap materials.

\section{Photochemistry in 3D photonic crystals}

The bandgap-induced enhancement of photochemical reactions has become an area of intense interest following recent pioneering studies by the Ozin group. As discussed above, at the band edge, the photon density of states is enhanced and the group velocity is reduced, which increases the effective optical path length in photonic crystals. The photons propagating with reduced velocity at the band edge are called slow photons. When a photochemical reaction activated by light at a wavelength corresponding to the band edge takes place in a photonic crystal, light adsorption and reaction efficiency are enhanced. Using this property of an inverse opal structure, an improvement in the photodegradation efficiency of organic pollutants has been demonstrated by Chen and co-workers [75]. The inverse opals, composed of amorphous titania nanocrystallites, were prepared by air plasma-assisted removal of polymeric opals filled with titania precursors, as shown in Figures $8(\mathrm{a}, \mathrm{b})$. Heat treatment was subsequently used to induce the phase transition to anatase, which exhibits photoactivity in the UV region. The photodegradation rates of methylene blue molecules adsorbed on the surfaces of inverse opals with various bandgap positions were evaluated according to the time of irradiation with monochromatic light centered at a wavelength of $370 \mathrm{~nm}$. As shown in Figure 8(c), an inverse opal with a bandgap of $345 \mathrm{~nm}$ produces the fastest degradation for normal incident light, attributable to the matching of the wavelengths of the irradiation light and the red band edge. The enhancement factor (EF), defined as the ratio of the decay rate constant of an inverse opal crystal to that of the corresponding fine powder, for an inverse opal with a bandgap of $370 \mathrm{~nm}$ was found to be less than unity, which is induced by the reflection of incident light due to the bandgap. Note also that the EFs for these inverse opals change when the angle of incidence of the light is off-normal, as shown in Figure 8(d). The inverse opal with a bandgap of $370 \mathrm{~nm}$ exhibits the highest EF (2.4) at an angle of incidence of $20^{\circ}$ due to the change in 

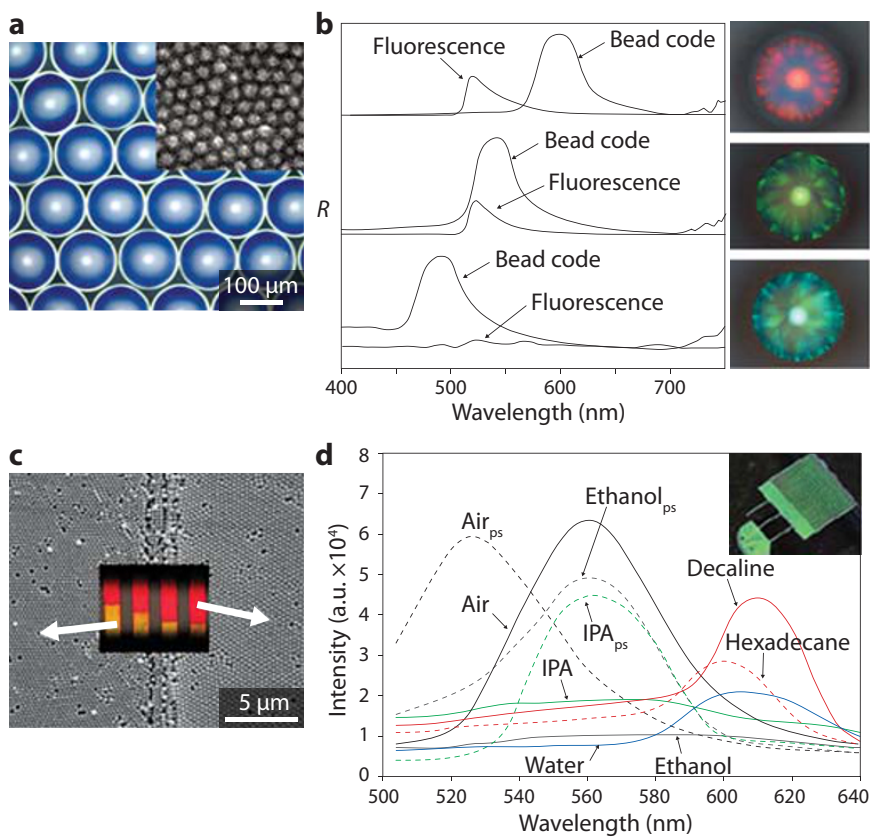

Figure 9. Encoded microcarriers and refractive index indicators. (a) Optical microscopy image of blue photonic microparticles. Inset shows the surface morphology of a microparticle. Adapted from Ref. 19 (๔ 2008 Wiley-VCH). (b) Reflectance and fluorescence spectra of three different microparticles and corresponding optical images. When human, mouse and rabbit lgG are immobilized on the red, green and blue photonic microparticles, respectively, only the red and green balls produce fluorescence after treatment with FITC-tagged anti-human and anti-rabbit IgG. Adapted from Ref. 86 (๔ 2006 Wiley-VCH). (c) Scanning electron microscopy image of hybrid colloidal crystals in the microchannel. Inset shows the corresponding optical microscopy image. (d) Reflectance spectra of two different colloidal crystals composed of silica particles (solid lines) and polystyrene particles (dotted lines). The crystal interstices are occupied with the indicated fluids. Inset shows an optical microscopy image of the colloidal crystal in the microfluidic channel. Adapted from Ref. 88 (@ 2006 RSC).

the stop-band position to $350 \mathrm{~nm}$, confirming the effect of slow photons on photodegradation. To assess the usefulness of such inverse opals, the same group investigated the effects of structural imperfections on these properties of inverse opals and found the photocatalytic enhancement by slow photons to be considerably tolerant of such imperfections [76]. In an advanced approach, the incorporation of platinum nanoparticles into a titania inverse opal backbone was found to enhance the EF to as high as 4.0 due to the extended lifetime of the UV-excited state in combination with the slow photon effect [77]. The slow photon effect of silica opal structures has also been employed in the photoisomerization of azobenzene molecules anchored on the surface of silica particles [78].

Light absorption efficiency can also be increased in semiconductor and photonic crystal bilayer systems. Nishimura et al. [79] prepared a dye-sensitized $\mathrm{TiO}_{2}$ photoelectrode coupled with a $\mathrm{TiO}_{2}$ inverse opal to enhance the photocurrent efficiency for solar-cell applications. The photonic crystal acts as a back-reflector at the bandgap, resulting in enhancement of the light path. In addition, the surface resonance mode at the interface between the photonic crystals and the semiconductor layer enhances the efficiency by an additional factor of up to $1.65[80,81]$.

\section{Biological tools}

The photonic bandgaps of colloidal crystals can be used as coding signals in biological suspension arrays. When colloidal crystals are prepared as microparticles suspended in a medium, the microparticles can carry specific information in the form of photonic bandgap positions. Therefore, such photonic microparticles can be used as biological arrays as an alternative to conventional planar arrays. Microparticle-based array systems have several advantages over planar arrays, including fast binding kinetics, large surface area to volume ratio, and inexpensive preparation.
To create photonic microparticles, the emulsion templating technique is used to achieve colloidal crystallization. When the emulsion droplets of the colloidal suspension are evaporated, the colloidal particles form close-packed crystals in a spherical shape [82-84]. In contrast, when particles with a repulsive interparticle potential are confined in the droplet, the particles form non-close-packed crystals without evaporation [85]. Microscopy images of blue microparticles prepared in this way are shown in Figures 9(a).

Zhao et al. [86] demonstrated biological screening using these photonic microparticles prepared to have three different colors. Red, green and blue photonic microparticles were prepared from monodisperse polymethylmethacrylate particles with diameters of $250 \mathrm{~nm}$, $220 \mathrm{~nm}$ and $210 \mathrm{~nm}$, and human, mouse and rabbit immunoglobulin $\mathrm{G}(\mathrm{IgG})$, respectively, were immobilized on the surfaces of photonic microparticles. When the mixture of microparticles was treated with fluorescein isothiocyanate-tagged anti-human $\operatorname{IgG}$ and anti-rabbit IgG, only the red and green photonic microparticles produced fluorescence, as shown in Figure 9(b). Microparticles coded with photonic bandgaps can thus be used in multiplex immunoassays [87]. The photonic bandgap is barely affected by fluorescence signals, making it possible to perform more precise analyses compared with those conducted using dye or quantum dot-tagged microparticles, which produce code signals that can interfere with the fluorescence.

The photonic bandgap can also be used in the detection of specific biomolecules or chemicals by exploiting the tunability of the bandgap in response to refractive index and lattice constant. One of the simplest approaches for this application involves the use of an opal structure built into a microfluidic channel as a refractive index indicator for fluids. When a liquid flows through a microfluidic channel occupied with colloidal crystals, the bandgap position varies with the refractive index of the fluid, allowing the refractive index to be measured by monitoring the reflectance spectrum of the colloidal crystals [88]. The blind region where the index of the colloids matches that of the liquid can be removed by fabricating two distinct regions in a single channel with silica and polystyrene colloidal crystals. Microscopy images and reflectance spectra for such hybrid colloidal crystals are shown in Figures 9(c,d).

There is a considerable history of research on advanced sensing units based on bulk colloidal crystals incorporating into the polymeric matrix binding sites for specific biomolecules. One of the pioneering works for photonic crystal-based sensors is that of Asher and co-workers [89,90]. They used PCCAs, which had been demonstrated to be useful as a practical colorimetric sensor for various types of analytes. As shown in Figure 10(a), the lattice constant of a PCCA can be increased or decreased through specific binding, in which the hydrogel network is modified to be capable of the molecular recognition of analytes [91-94]. Such binding events result in a considerable difference between the ionic concentrations of the hydrogel and the surrounding medium, which in turn results in the swelling of the hydrogel by osmotic pressure and in a red-shift in the bandgap position. When analytes have two or more binding groups, the hydrogel network can be compressed, resulting in a blue-shift in the bandgap position. The swelling behavior is highly reversible and can be observed directly by monitoring the color change. Such a mechanism can, for example, allow the concentration of glucose in the eye to be monitored in real time by fabricating contact lenses out of this PCCA for patients suffering from diabetes [91,93,94].

Zhao et al. [95] prepared inverse opaline microparticles with hydrogel networks for label-free DNA detection. As shown in the schematic diagram in Figure 10(b), the incorporation of probe DNA into the hydrogel network enables the detection of specific DNA through the blue-shift of the reflection peak. The hybridization of target DNA with complementary base pairs reduces the grid size of the hydrogel network by forming a stiff double-helix structure. An increase in the concentration of the target DNA thus results in an increase in the shrinkage of the hydrogel and an increase in the blue-shift.

Molecular imprinting technique has been used to create an artificial antibody with complementary shape and binding sites in a colloidal 
a

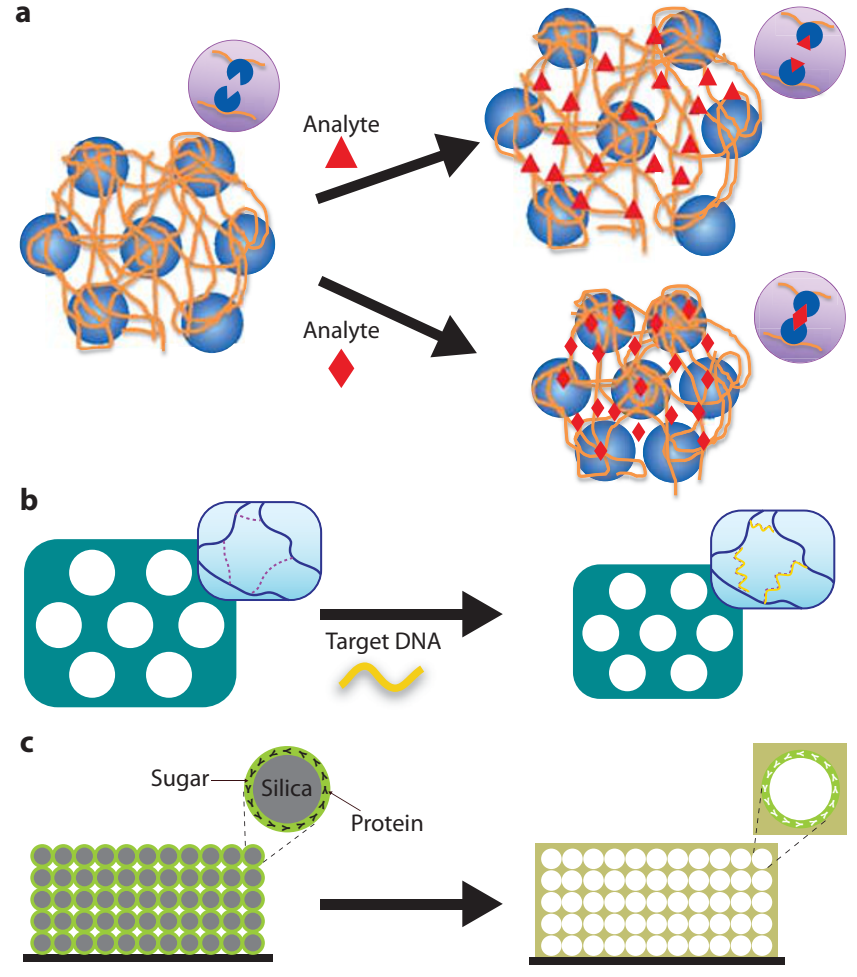

Figure 10. Biosensors based on lattice constant changes. (a) Schematic diagram of an intelligent polymerized crystalline colloidal array for sensing analyte molecules. The adsorption of the analyte induces swelling and a red-shift in the bandgap or constriction and blue-shift in the bandgap. (b) DNA sensors based on inverse opal structures. The hybridization of target DNA reduces the lattice constant and results in a blue-shift of the spectrum. (c) Molecular imprinting of a protein into an inverse opal film to produce an artificial antibody that has complementary shape and binding sites.

crystal platform [96,97]. As illustrated in Figure 10(c), specific proteins can be imprinted into nanocavities in an inverse opal structure through the selective removal of silica opal and proteins from a composite consisting of hydrogel and a protein-filled opal film. The resulting hierarchical porous photonic crystals undergo a red-shift in the peak position due to swelling upon molecular recognition of imprinted protein molecules based on the shapes of the nanocavities.

\section{Summary and outlook}

Over the past few decades, several different approaches have been employed to produce colloidal nanostructures in investigations of photonic applications. This research, conducted by many different research groups, has expanded the range of applications to include nonphotonic materials, which might result for instance in the convergence of photonics and biomedical applications and in energy-harvesting devices. In particular, colloidal crystals and composite and inverse opals have been investigated and modified for specific applications. In spite of their limited optical response, or in other words their incomplete pseudo-photonic bandgap, they can be successfully implemented in relatively simple applications. By adding functional molecules into such colloidal nanostructures, photonic crystal-based sensor devices have been developed and their technological level has in some cases matured close to the point of commercialization or the opening of new markets. New types of photonic nanostructures, such as photonic glasses, have been introduced for angle-independent light reflection, random lasing and other applications.

In some original applications of photonic crystals, several research groups have fabricated optical circuits out of colloidal particles and demonstrated their great potential [74]. However, it is still difficult to fabricate defect-free colloidal crystals and then to create prescribed defects because of the inherent defects that arise during self-assembly. As a result, research activity in this area has gradually decreased. However, in a new approach, the use of selective chemical glue on particle surfaces such as DNA or peptides has been proposed [98]. Further developments might enable the fabrication of better photonic devices based on colloidal particles. Moreover, recent progress in the synthesis of nonspherical particles is accelerating research into the colloidal crystallization of new crystal phases [99]. One promising strategy with strong potential for producing new photonic properties is the use of directional interparticle interaction with chemical patterns on a colloid surface to create and design new types of colloidal lattices.

\section{Acknowledgments}

This work was supported by a grant from the Creative Research Initiative Program of the Ministry of Education, Science and Technology (MEST), Korea, for research on 'Complementary hybridization of optical and fluidic devices for integrated optofluidic systems'. The authors also appreciate partial support from the Brain Korea 21 Program. Gi-Ra Yi is thankful for the support of a MEST National Research Foundation grant (No. 2009-0082451), an International Collaboration grant (No. Sunjin-2010-002) from the Korean Ministry of Knowledge Economy and funding under the R\&D Program of the National Fusion Research Institute of Korea. The authors also acknowledge discussions with Andy Hollingsworth and Mark T. Elsesser.

\section{References}

1. S. G. Johnson, J. D. Joannopoulos, Photonic Crystrals: The Road from Theory to Practice (Springer, USA, 2001).

2. M. Qi et al., Nature 429, 538 (2004).

3. S.-H. Kim, J.-M. Lim, S.-K. Lee, C.-J. Heo, S.-M. Yang, Soft Matter. 6, $1092(2010)$

4. Y. Kang, J. J. Walish, T. Gorishnyy, E. L. Thomas, Nat. Mater. 6, 957 (2007).

5. E. Kuramochi, M. Notomi, T. Kawashima, J. Takahashi, Opt. Quant. Elect. 34, 53 (2002).

6. M. Campbell, D. N. Sharp, M. T. Harrison, R. G. Denning, A. J. Turberfield, Nature 404, 53 (2000).

7. F. Marlow, Muldarisnur, P. Sharifi, R. Brinkmann, C. Mendive, Angew. Chem. Int. Ed. 48, 6212 (2009).

8. J. F. Calisteo-Lopez et al., Adv. Mater. 23, 30(2010).

9. J. W. Vanderhoff, J. F. Vitkuske, E. B. Bradford, T. Alfrey, J. Polym. Sci. 20, 225 (1956).

10. W. B. Russel, D. A. Saville, W. R. Schowalter, Colloidal Dispersions (Cambridge University Press, USA, 1989)

11. C. P. Royall, M. E. Leunissen, A. van Blaaderen, J. Phys.: Condens. Matter 15, S3581 (2003).

12. A. Yethiraj, A. van Blaaderen, Nature 421, 513 (2003).

13. S. H. Foulger et al., Adv. Mater. 13, 1898 (2001).

14. R. Lawrence, Y. Ying, P. Jiang, S. H. Foulger Adv. Mater. 18, 300 (2006).

15. J. M. Weissman, H. B. Sunkara, A. S. Tse, S. A. Asher, Science 274, 959 (1996).

16. Y.-J. Lee, P. V. Braun, Adv. Mater. 15, 563 (2003).

17. H. Yang, P. Jiang, Langmuir 26, 13173 (2010).

18. P. Jiang, M. J. McFarland, J. Am. Chem. Soc. 126, 13778 (2004).

19. S.-H. Kim et al., Adv. Mater. 20, 1649 (2008).

20. G. Subramanian, V. N. Manoharan, J. D. Thorne, D. J. Pine, Adv. Mater. 11, 1261 (1999).

21. P. Jiang, J. F. Bertone, K. S. Hwang, V. L. Colvin, Chem. Mater. 11, 2132 (1999).

22. J. E. G. J. Wijnhoven, W. L. Vos, Science 281, 802 (1998).

23. O. D. Velev, T. A. Jede, R. F. Lobo, A. M. Lenhoff, Nature 389, 447 (1997).

24. B. Hatton, L. Mishchenko, S. Davis, K. H. Sandhage, J. Aizenberg, Proc. Natl Acad. Sci. 107, 10354 (2010).

25. E. Hecht, A. Zajac, Optics, 4th Ed. (Addison Wesley, USA, 2002).

26. Y. Nevez-Oz, M. Golosovsky, D. Davidov, J. Appl. Phys. 95, 5989 (2004).

27. G. Pan, A. K. Sood, S. A. Asher, J. Appl. Phys. 84, 83 (1998).

28. P. A. Rundquist, P. Photinos, S. Jagannathan, S. A. Asher, J. Chem. Phys. 91, 4932 (1989).

29. P. Wochner et al., Proc. Natl Acad. Sci. 106, 11511 (2009).

30. S. Gottardo et al., Nat. Photonics 2, 429 (2008).

31. P. D. García, R. Sapienza, Á. Blanco, C. López, Adv. Mater. 19, 2597 (2007).

32. B. Q. Dong et al., Opt. Express 18, 14430 (2010).

33. M. Harun-Ur-Rashid et al., ChemPhysChem, 11, 579 (2010). 
34. J. Bertolotti et al., Adv. Func. Mater. 20, 965 (2010).

35. D. S. Wiersma, Nat. Phys. 4, 359 (2008).

36. K. Ueno, A. Inaba, Y. Sano, M. Kondoh, M. Watanabe, Chem. Commun., 3603 (2009).

37. P. D. García, R. Sapienza, C. López, Adv. Mater. 22, 12 (2010).

38. S.-K. Lee, G.-R. Yi, J. H. Moon, S.-M. Yang, D. J. Pine, Adv. Mater. 18, 2111 (2006).

39. A. C. Arsenault, D. P. Puzzo, I. Manners, G. A. Ozin, Nat. Photonics 1, 468 (2007).

40. S.-H. Kim, S.-J. Jeon, W. C. Jeong, H. S. Park, S.-M. Yang, Adv. Mater. 20, 4129 (2008).

41. I. Lee et al., Adv. Mater. 22, 4973(2010).

42. E. Kim, Y. Xia, G. M. Whitesides, Adv. Mater. 8, 245 (1996).

43. S. M. Yang, H. Miguez, G. A. Ozin, Adv. Funct. Mater. 12, 425 (2002),

44. S.-H. Kim, H. S. Park, J. H. Choi, J. W. Shim, S.-M. Yang, Adv. Mater. 22, 946 (2010).

45. Z.-Z. Gu, A. Fujishima, O. Sato, Angew. Chem. Int. Edit. 41, 2067 (2002).

46. S. Y. Lee, S.-H. Kim, C.-J. Heo, H. Hwang, S.-M. Yang, Phys. Chem. Chem. Phys. 12, 11861 (2010).

47. H. Kim et al., Nat. Photonics 3, 534 (2009).

48. H.-Y. Ko, J. Park, H. Shin, J. Moon, Chem. Mater. 16, 4212 (2004).

49. J. Park, J. Moon, H. Shin, D. Wang, M. Park, J. Colloid Interf. Sci. 298, 713 (2006).

50. K. Burkert et al., Langmuir 23, 3478 (2007).

51. S.-H. Kim, J.-M. Lim, W. C. Jeong, D.-G. Choi, S.-M. Yang, Adv. Mater. 20, 3211 (2008).

52. Y. Masuda, T. Itoh, K. Koumoto, Adv. Mater. 17, 841 (2005).

53. S.-H. Kim, S.-H. Kim, S.-M. Yang, Adv. Mater. 21, 3771 (2009).

54. X. Xu, G. Friedman, K. D. Humfeld, S. A. Majetich, S. A. Asher, Adv. Mater. 13, 1681 (2001).

55. J. Ge, Y. Hu, Y. Yin, Angew. Chem. Int. Edit. 46, 7428 (2007).

56. J. Ge, Y. Yin, Adv. Mater. 20, 3485 (2008).

57. D. P. Puzzo, A. C. Arsenault, I. Manners, G. A. Ozin, Angew. Chem. Int. Edit. 48, 943 (2009).

58. K. Ueno, J. Sakamoto, Y. Takeoka, M. Watanabe, J. Mater. Chem. 19, 4778 (2009).

59. T. S. Shim, S.-H. Kim, J. Y. Sim, J.-M. Lim, S.-M. Yang, Adv. Mater. 22, 4494 (2010).

60. J. Ge et al., J. Am. Chem. Soc. 131, 15687 (2009).

61. J. D. Forster et al., Adv. Mater. 22, 2939 (2010).

62. O. L. J. Pursiainen et al., Opt. Express 15, 9553 (2007).

63. P. Bartlett, A. I. Campbell, Phys. Rev. Lett. 95, 128302 (2005).

64. M. E. Leunissen et al., Nature 437, 235 (2005).
65. A.-P. Hynninen, J. H. J. Thijssen, E. C. M. Vermolen, M. Dijkstra, A. van Blaaderen, Nat. Mater. 6, 202 (2007).

66. M. N. Shkunov et al., Adv. Funct. Mater. 12, 21 (2002).

67. H. Yamada, T. Nakamura, Y. Yamada, K. Yano, Adv. Mater. 21, 4134 (2009).

68. S.-H. Kim, S.-H. Kim, W. C. Jeong, S.-M. Yang, Chem. Mater. 21, 4993 (2009).

69. M. Scharrer, A. Yamilov, X. Wu, H. Cao, R. P. H. Chang, Appl. Phys. Lett. 88, 201103 (2006).

70. S. Furumi, H. Fudouzi, H. T. Miyazaki, Y. Sakka, Adv. Mater. 19, 2067 (2007).

71. J. R. Lawrence, Y. Ying, P. Jiang, S. H. Foulger, Adv. Mater. 18, 300 (2006).

72. A. Blanco et al., Nature 405, 437 (2000).

73. Y. A. Vlasov, X.-Z. Bo, J. C. Sturm, D. J. Norris, Nature 414, 289 (2001).

74. S. A. Rinne, F. G.-Santama ría, P. V. Braun, Nat. Photonics 2, 52 (2007).

75. J. I. L. Chen, G. von Freymann, S. Y. Choi, V. Kitaev, G. A. Ozin, Adv. Mater. 18, 1915 (2006).

76. J. I. L. Chen, G. von Freymann, V. Kitaev, G. A. Ozin, J. Am. Chem. Soc. 129, 1196 (2007).

77. J. I. L. Chen, E. Loso, N. Ebrahim, G. A. Ozin, J. Am. Chem. Soc. 130, 5420 (2008).

78. J. I. L. Chen, G. A. Ozin, Adv. Mater. 20, 4784 (2008).

79. S. Nishimura et al., J. Am. Chem. Soc. 125, 6306 (2003).

80. P. G. O'Brien et al., Adv. Mater. 19, 4177 (2007).

81. P. G. O'Brien et al., Adv. Mater. 20, 1577 (2008).

82. O. D. Velev, A. M. Lenhoff, E. W. Kaler, Science 287, 2240 (2000).

83. G.-R. Yi et al., Adv. Mater. 14, 1137 (2002).

84. S.-H. Kim, S. Y. Lee, G.-R. Yi, D. J. Pine, S.-M. Yang, J. Am. Chem. Soc. 128, 10897 (2006).

85. S.-H. Kim, S.-J. Jeon, S.-M. Yang, J. Am. Chem. Soc. 130, 6040 (2008).

86. X. Zhao et al., Angew. Chem. Int. Edit. 45, 6835 (2006).

87. Y. Zhao et al., Anal. Chem. 80, 1598 (2008).

88. S.-K. Lee, G.-R. Yi, S.-M. Yang, Lab Chip 6, 1171 (2006).

89. S. A. Asher, US Patent 4627689 (1986).

90. J. H. Holtz, S. A. Asher, Nature 389, 829 (1997).

91. S. A. Asher et al., J. Am. Chem. Soc. 125, 3322 (2003).

92. A. C. Sharma et al., J. Am. Chem. Soc. 126, 2971 (2004).

93. M. Ben-Moshe, V. Alexeev, S. A. Asher, Anal. Chem. 78, 5149 (2006).

94. D. Nakayama, Y. Takeoka, M. Watanabe, K. Kataoka, Angew. Chem. Int. Edit. 42, 4197 (2003)

95. Y. Zhao et al., Adv. Funct. Mater. 20, 976 (2010).

96. X. Hu, G. Li, J. Huang, D. Zhang, Y. Qiu, Adv. Mater. 19, 4327 (2007).

97. Y.-J. Zhao et al., Angew. Chem. Int. Edit. 48, 7350 (2009).

98. A. J. Kim, R. Scarlett, P. L. Biancaniello, T. Sinno, J. C. Crocker, Nat. Mater. 8, 52 (2009).

99. S.-M. Yang, S.-H. Kim, J.-M. Lim, G.-R. Yi, J. Mater. Chem. 18, 2177 (2008).

\section{Author profiles}

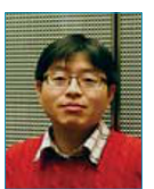

\section{Shin-Hyun Kim}

Shin-Hyun Kim received his BS degree in chemical engineering from Yonsei University, Korea, in 2004 and a PhD degree in chemical and biomolecular engineering from KAIST, Korea, in 2009. He is currently working as a postdoctoral researcher at Harvard University, USA. His major research interests include colloidal self-organization in confined geometry, droplet-based microfluidics and synthesis of functional microparticles and capsules.

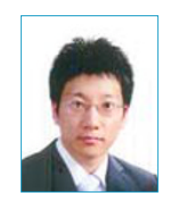

\section{Su Yeon Lee}

Su Yeon Lee is a PhD candidate in chemical and biomolecular engineering at KAIST, Korea. He received his BS degree in chemical engineering from Yonsei University, Korea, in 2005, and an MS degree in chemical and biomolecular engineering from KAIST in 2007. His current research interest is in plasmonic nanostructures for biomolecular sensors as well as the fabrication of photonic microparticles.

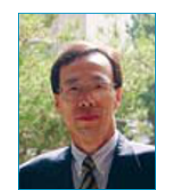

\section{Seung-Man Yang}

Seung-Man Yang received a PhD degree in chemical engineering from Caltech, USA, in 1985. He subsequently joined KAIST, Korea, as a professor of chemical and biomolecular engineering. He has served KAIST as a director of the Computing Center and as a Department Chair. He currently leads the Creative Research Initiative Center for Integrated Optofluidic Systems. His principal contributions have been in theories and experimental methods for fabricating ordered macrocrystalline structures, which can be applied as innovative functional nanoscopic materials such as optoelectronic devices and biosensors.

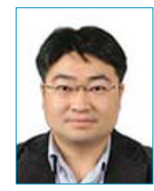

\section{Gi-Ra Yi}

Gi-Ra Yi received his BS degree in chemical engineering from Yonsei University, Korea, in 1997 and MS (1999) and PhD (2003) degrees in chemical and biomolecular engineering from KAIST, Korea. He undertook postdoctoral research at the University of California, Santa Barbara, USA, and worked briefly for the Corporate R\&D Center of LG Chem Research Park and the Korea Basic Science Institute as a research scientist. In 2009, he joined Chungbuk National University, Korea, as an assistant professor in engineering chemistry. His current interest is in self-assemblies of colloidal particles at micrometer or nanometer scales, as well as multiphase microfluidics. 